\title{
Effects of structural flexibility of wings in flapping flight of butterfly
}

\section{$\operatorname{AUTHOR}(S)$ :}

Senda, Kei; Obara, Takuya; Kitamura, Masahiko;

Yokoyama, Naoto; Hirai, Norio; lima, Makoto

\section{CITATION:}

Senda, Kei ...[et al]. Effects of structural flexibility of wings in flapping flight of butterfly. Bioinspiration \& biomimetics 2012, 7(2): 025002.

ISSUE DATE:

2012-06

URL:

http://hdl.handle.net/2433/157349

\section{RIGHT:}

@ 2012 IOP Publishing Ltd.; この論文は出版社版でありません。引用の 際には出版社版をご確認ご利用ください。; This is not the published version. Please cite only the published version. 


\title{
Effects of Structural Flexibility of Wings in Flapping Flight of Butterfly
}

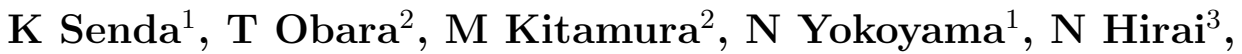 \\ and $\mathrm{M}$ Iima $^{4}$ \\ ${ }^{1}$ Kyoto University, Yoshida-Honmachi, Sakyo-ku, Kyoto 606-8501, Japan \\ ${ }^{2}$ Kanazawa University, Kakuma-machi, Kanazawa, Ishikawa 920-1192, Japan \\ ${ }^{3}$ Osaka Prefecture University, 1-1 Gakuen-cho, Nakaku, Sakai, Osaka 599-8531, \\ Japan \\ ${ }^{4}$ Hiroshima University, 1-7-1 Kagamiyama, Higashi-Hiroshima, Hiroshima 739-8521, \\ Japan \\ E-mail: senda@kuaero.kyoto-u.ac.jp
}

\begin{abstract}
The objective of this paper is to clarify the effects of structural flexibility of wings of a butterfly in flapping flight. For this purpose, a dynamics model of a butterfly is derived by Lagrange's method, where the butterfly is considered as a rigid multi-body system. The panel method is employed to simulate the flow field and the aerodynamic forces acting on the wings. The mathematical model is validated by agreement of the numerical result with the experimentally measured data. Then, periodic orbits of flapping-of-wings flights are parametrically searched in order to fly the butterfly models. Almost periodic orbits are found, but they are unstable. Deformation of the wings is modeled in two ways. One is bending and its effect on the aerodynamic forces is discussed. The other is passive wing torsion caused by structural flexibility. Numerical simulations demonstrate that the flexible torsion reduces the flight instability.
\end{abstract}

Submitted to: Bioinspiration $\mathscr{E}$ Biomimetics 


\section{Introduction}

Butterflies can maintain desired flapping-of-wings flights stably against environmental uncertainties and variations such as gust, weight gain or loss etc., by their adaptationcapability, whereas they cannot repeat the same movement with sufficient accuracy. This study discusses the principle that realizes flapping-of-wings flights which are rhythmical periodic motion.

There are many unknowns for the stable flight, for example, "how the butterfly moves," and "how the control is realized." Concretely, a research project investigates these issues by the biological analysis through experimental observations of living butterflies and by the systems engineering or synthetic approach [1-7]. One of the obtained results is that the free vortices in the wakes stabilize the flight. Following these studies, stabilization due to the structural flexibly of the wings is demonstrated.

There are some researches relating deformable wings of insects. Vanella et al. [8] studied numerically using a deformable wing model composed of two links articulated by a rotary joint. Their 2-dimensional numerical simulations show that the elastic deformation of the wings sometimes enhances the aerodynamic performance. Walker et al. [9, 10] and Young et al. [11] measured deformation of wings of a desert locust through a stroke, and showed that the camber and twist enhance aerodynamic efficiency. Tanaka and Shimoyama [12] made a butterfly ornithopter that can fly for a few periods without feedback control of the flapping motion. However, the effects of deformable wings of butterflies have not discussed enoughly, especially the stability of flight dynamics have not clarified yet. Further study is needed. Therefore this study discusses two different effect of wing deformation as follows, i.e. effects on aerodynamic forces and effects on flight stability.

A mathematical model of a butterfly is explained in section 2. The mathematical model of a flapping butterfly is constructed for numerical simulations. In the modeling, the butterfly is considered as a rigid multi-body system, and its wings is flat without deformation. Aerodynamic forces applied to the wings are formulated by the panel method [3-5] where the influence of the unsteady wakes can be incorporated. This model is referred as the basic model in this paper. The experimental observation of living butterflies is also outlined in section 2. Observation experiments are conducted using an experimental system with a low-speed wind tunnel, and the motions and aerodynamic forces applied to the living butterflies are measured quantitatively. The flapping motion of a free-flying butterfly is also measured by the experimental system, where the trajectory of flapping flight, i.e. the trajectory of the thorax and joints is measured. The validity and accuracy of the numerical model are examined by comparing the calculated aerodynamic forces with the measured experimental data. However, the flapping flight does not continue stably even if the numerical model repeats the measured joint trajectory.

Hence in section 3, periodic orbits of flapping-of-wings flights are searched in order to fly the butterfly model, and almost periodic orbits are obtained. The butterfly 
can fly for several periods when one of those motions is just repeated, whereas no feedback control is applied. It is reported that the unsteady wake-induced flow has a type of feedback stabilization effect that enables the butterfly to fly several periods $[3,5]$. This is the feedback stabilization effect brought by this system through the dynamic characteristic of the flow field, and it can be considered as an implicit control. However, the flapping flights are not sufficiently stabilized only by this effect, and the numerical model oscillates and goes down after a several flapping motions.

In section 4, experiments are conducted again using tethered actual butterflies, where wing deformation is observed, and the necessity of introducing the wing deformation into the basic model is presented. Structurally flexible wings of the actual butterflies are passively deformed by the aerodynamic forces applied to the wings. The above model is called the time-varying camber model that describes the measured deformation as a function of time. This time-varying camber is introduced into the basic model, and the aerodynamic forces are calculated. The effects of the wing deformation on the aerodynamic forces using this model are quantitatively evaluated. In the section, the wing deformations of a tethered butterfly are used to maintain measurement accuracy. The time-varying camber model is compared with the basic model whose wings are always flat. The aerodynamic effects of the wing deformation is quantified by using the experimental observation and the models. It will be shown that the aerodynamic forces in the time-varying camber model agrees better with that in the experiment than that in the basis model. It is based on the measured deformation and is the same way that Young et al. [11] did for the desert locust.

In section 5, it is clarified that the effects of the passive deformation to the flight stability.

The wings of the time-varying camber model are not passively deformed by aerodynamic forces applied to the wings because the wing deformation is given as a function of time. In case of the structurally flexible wings of actual butterflies, the wing motion determines the flow field around the wings, and the aerodynamic forces applied to the wings determine the elastic deformation of the wings. This is a fluid-structure interaction problem. To determine the wing deformation accurately by solving the problem, a precise model of wing stiffness distribution is needed. It is almost impossible to calculate the deformation accurately because this precise modeling is more difficult than the wing deformation measurement.

As the typical modeling of the deformation, only wing torsion is modeled [13-15] when the measurements of the wing deformation is inaccurate. In this study, a passive torsion model is introduced where a flat wing is passively twisted in accordance with the aerodynamic disturbance. The joint trajectory of the basic model can be controlled to the desired trajectory without error, whereas that of actual butterflies cannot be controlled as well because their wings are twisted by various unexpected disturbance. The passive torsion model is compared with the basic model whose wings always track the planned trajectory. It will be shown that the passive wing deformation increases the stability of flapping flight. 
Sections 3 and 5 deal with the flight dynamics of butterflies that discuss the stabilizing effects introduced by the implicit controls. The model in this study will be agree well with experimental results in section 2, whereas both of the rigid multibody model in sections 3 and the passive torsion model in section 5 are unstable in terms of flight dynamics. Hence, a living butterfly stabilizes its flight by an active control though its flight dynamics may be unstable. Taylor and Zbikowski [16] also consider that the flight dynamics of the desert locust is unstable and is stabilized by an active control. It is reasonable that the model is dynamically unstable if the modeled original system is unstable. In this situation, it is difficult to predict the behavior of original system qualitatively by using the model. If the model and/or the initial state has an even small error from those of the original system, the error increases in time because of the instability. Therefore, this study will not obtain quantitative results but qualitative results. It is that the stability of the flapping butterfly is increased by the unsteady wake induced flow in section 3 and by the passive wing torsion in section 5 .

Finally, concluding remarks are given in section 6 .

\section{Model and experiments of butterfly}

\subsection{Outline of flapping mechanism}

Fig. 1 schematically illustrates a flapping mechanism of insects that is driven by indirect muscles [17]. The wings are connected to the thorax by lever mechanisms, where the wings have been evolved from exoskeleton. The end of the wing link is connected to the lateral suctum by a hinge in the thorax. The wing link is also connected to the thoracic wall in the thorax by the other hinge next to the end hinge. When the suctum lifts up, the principle of leverage moves the wing down. The horizontal muscles connecting the front wall to the posterior wall of the thorax, which are known as dorsal longitudinal muscles, tends to contract and increase the curvature of the suctum. The butterflies have vertical muscles known as the dorsoventral muscles running from the roof of the thorax to the floor. Contraction of the dorsoventral muscles pulls the suctum down. While relaxation of the dorsoventral muscles results in the dorsal longitudinal muscles' contraction and the suctum is pushed up. This flapping mechanism can be considered as almost one degree-of-freedom vibrating mechanism. A butterfly contracts the dorsoventral muscles periodically to vibrate the mechanism for flapping of the wings. According to experimental observations in a later section, each butterfly has an almost unique flapping eigenfrequency. It may be because to vibrate sympathetically at eigenfrequency of the mechanism realizes efficient flapping motion [18]. As suggested in the anatomical observation below, the other direct muscles may add slight motions, which are in the extent of modification to the basic vibration. 

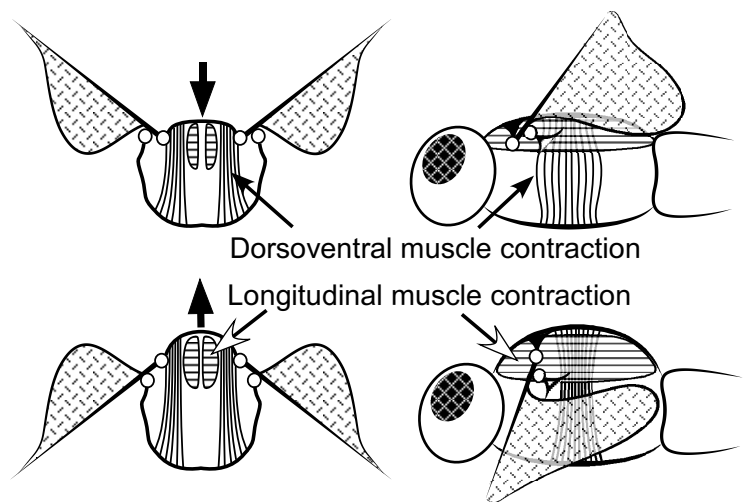

Figure 1. Indirect muscle system driving wing mechanism (left: thorax cross section, right: thorax longitudinal section)

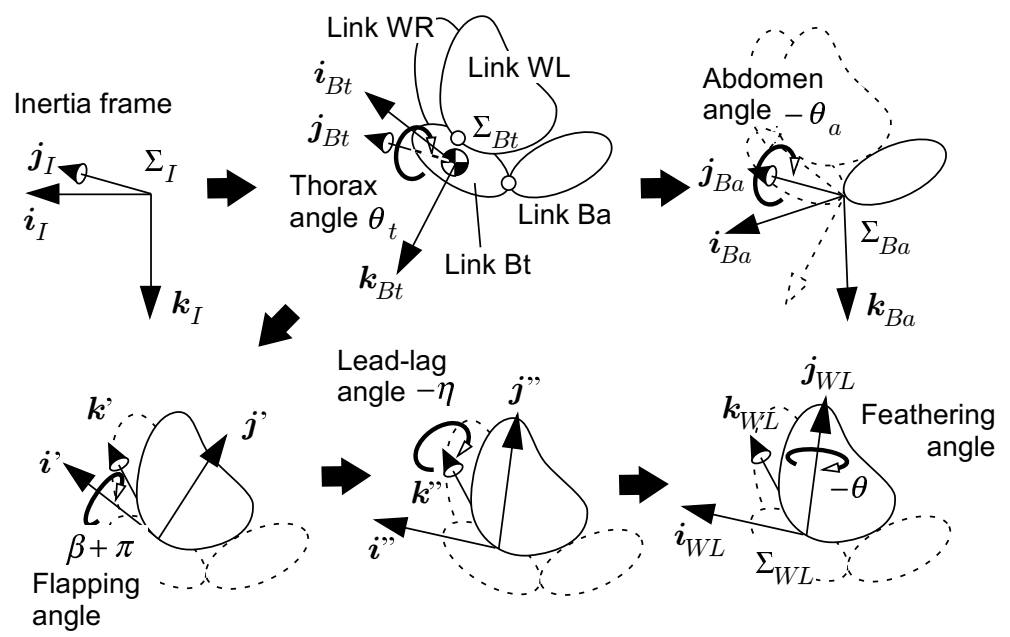

Figure 2. Frames and coordinates for modeling of a butterfly.

\subsection{Dynamics model of butterfly as rigid multi-body system}

A butterfly is modeled by a rigid multi-body system in the same way as typical robot modeling, which is simple dynamics modeling. The model defined in this section is refereed as the basic model. In later sections, it will be modified so as to consider wings' deformation because experimental observations have found that butterfly wings are elastically deformed by aerodynamic forces.

The butterfly model is a multi-body system with 4 links as shown in Fig. 2, which is composed of the thorax Bt considered as the main body, the abdomen Ba, the left wings WL, and the right wings WR. Both the model and its motions are supposed to be symmetric bilaterally. A pair of fore and hind wings on each side is modeled by a plate as shown in Fig. 2. The joint between the thorax and wing has 3 rotational degrees of freedom (DOF) and the joint between the thorax and the abdomen has $1 \mathrm{DOF}$.

The kinematics of the butterfly is described by the Lagrangian formalism,

$$
\frac{\mathrm{d}}{\mathrm{d} t}\left(\frac{\partial L}{\partial \dot{\boldsymbol{\theta}}}\right)-\frac{\partial L}{\partial \boldsymbol{\theta}}=\boldsymbol{\tau}
$$


The Lagrangian $L=K-V$ is composed of the kinetic energy $K=\frac{1}{2} \dot{\boldsymbol{\theta}}^{T} \boldsymbol{M}(\boldsymbol{\theta}) \dot{\boldsymbol{\theta}}$ and the potential energy due to gravity $V$. The generalized coordinates are divided into the thorax coordinates denoted by the subscript 1 and the joint coordinates by the

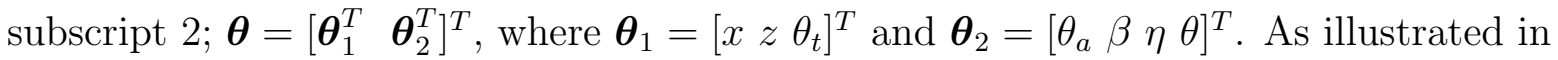
Fig. $2, x, z$, and $\theta_{t}$ are the $x, z$-positions and the attitude angle of the thorax, $\theta_{a}$ the abdomen angle, $\beta$ the flapping angle in up-down direction, $\eta$ the lead-lag angle, and $\theta$ the feathering angle representing a torsion angle. The generalized forces are also divided into $\boldsymbol{\tau}=\left[\begin{array}{ll}\boldsymbol{\tau}_{1}^{T} & \boldsymbol{\tau}_{2}^{T}\end{array}\right]^{T}$, where $\boldsymbol{\tau}_{1}$ and $\boldsymbol{\tau}_{2}$ corespond to $\boldsymbol{\theta}_{1}$ and $\boldsymbol{\theta}_{2}$, respectively. Note that the inertial matrix $\boldsymbol{M}$ depends on time through $\boldsymbol{\theta}$. The generalized force $\boldsymbol{\tau}$ consists of the aerodynamic force $\boldsymbol{\tau}_{\text {air }}$ obtained from the forces $\boldsymbol{f}_{\text {air }}$ according to the panel method developed in the following sections and the control force $\boldsymbol{\tau}_{\text {cont }}$ applied to the thorax and the joints. The Lagrangian equation of the motion is rewritten as

$$
\boldsymbol{M} \ddot{\boldsymbol{\theta}}+\dot{\boldsymbol{M}} \dot{\boldsymbol{\theta}}-\frac{1}{2} \frac{\partial}{\partial \boldsymbol{\theta}}(\dot{\boldsymbol{\theta}} \boldsymbol{M} \dot{\boldsymbol{\theta}})+\frac{\partial V}{\partial \boldsymbol{\theta}}=\boldsymbol{\tau}_{\text {air }}+\boldsymbol{\tau}_{\text {control }}
$$

By dividing the variables into the thorax part denoted by the subscript 1 and the joint parts by the subscript 2, Eq. (2) can symbolically be rewritten as

$$
\left[\begin{array}{ll}
\boldsymbol{M}_{11} & \boldsymbol{M}_{12} \\
\boldsymbol{M}_{21} & \boldsymbol{M}_{22}
\end{array}\right]\left[\begin{array}{l}
\ddot{\boldsymbol{\theta}}_{1} \\
\ddot{\boldsymbol{\theta}}_{2}
\end{array}\right]+\left[\begin{array}{l}
\boldsymbol{h}_{1} \\
\boldsymbol{h}_{2}
\end{array}\right]=\left[\begin{array}{l}
\boldsymbol{\tau}_{\text {cont } 1} \\
\boldsymbol{\tau}_{\text {cont } 2}
\end{array}\right]
$$

where $\boldsymbol{h}=\left[\begin{array}{ll}\boldsymbol{h}_{1}^{T} & \boldsymbol{h}_{2}^{T}\end{array}\right]^{T}=\dot{\boldsymbol{M}} \dot{\boldsymbol{\theta}}-\frac{1}{2} \frac{\partial}{\partial \boldsymbol{\theta}}(\dot{\boldsymbol{\theta}} \boldsymbol{M} \dot{\boldsymbol{\theta}})+\frac{\partial V}{\partial \boldsymbol{\theta}}-\boldsymbol{\tau}_{\text {air }}$.

In every simulation, $\boldsymbol{\theta}_{2}$ is given as a desired trajectory $\boldsymbol{\theta}_{2 \mathrm{~d}}(t)$ of time $t$. In a tethered simulation, $\boldsymbol{\theta}_{1}$ is constant in time since the thorax is fixed. In a free-flight situation, $\boldsymbol{\tau}_{\text {cont1 }}$ is zero because the butterfly cannot directly apply force to the thorax. In the following section, the aerodynamic forces $\boldsymbol{f}_{\text {air }}$ and the generalized forces $\boldsymbol{\tau}_{\text {air }}$ are obtained by the panel method. Then, the desired motions in both cases are realized by the following $\boldsymbol{\tau}_{\text {cont1 }}$ and $\boldsymbol{\tau}_{\text {cont2 }}$.

In the tethered simulation, the acceleration of thorax is $\ddot{\boldsymbol{\theta}}_{1}=\mathbf{0}$ and

$$
\begin{aligned}
& \boldsymbol{\tau}_{\text {cont } 1}=\boldsymbol{M}_{12} \ddot{\boldsymbol{\theta}}_{2 \mathrm{~d}}+\boldsymbol{h}_{1} \\
& \boldsymbol{\tau}_{\text {cont } 2}=\boldsymbol{M}_{22} \ddot{\boldsymbol{\theta}}_{2 \mathrm{~d}}+\boldsymbol{h}_{2}
\end{aligned}
$$

where $\boldsymbol{\tau}_{\text {cont1 }}$ is applied to the thorax as the constraint force to maitain $\ddot{\boldsymbol{\theta}}_{1}=\mathbf{0}$.

In the free-flight situation,

$$
\begin{aligned}
& \boldsymbol{\tau}_{\text {cont } 1}=\mathbf{0} \\
& \boldsymbol{\tau}_{\text {cont } 2}=\boldsymbol{M}_{21} \ddot{\boldsymbol{\theta}}_{1}+\boldsymbol{M}_{22} \ddot{\boldsymbol{\theta}}_{2 \mathrm{~d}}+\boldsymbol{h}_{2}
\end{aligned}
$$

where $\ddot{\boldsymbol{\theta}}_{1}=-\boldsymbol{M}_{11}^{-1}\left(\boldsymbol{M}_{12} \ddot{\boldsymbol{\theta}}_{2 \mathrm{~d}}+\boldsymbol{h}_{1}\right)$.

\subsection{Panel method model for aerodynamics}

In the flight of the butterfly, there is a feature in which the flapping frequency is small [18] as well as small Reynolds number. Moreover, the relative velocity of the 


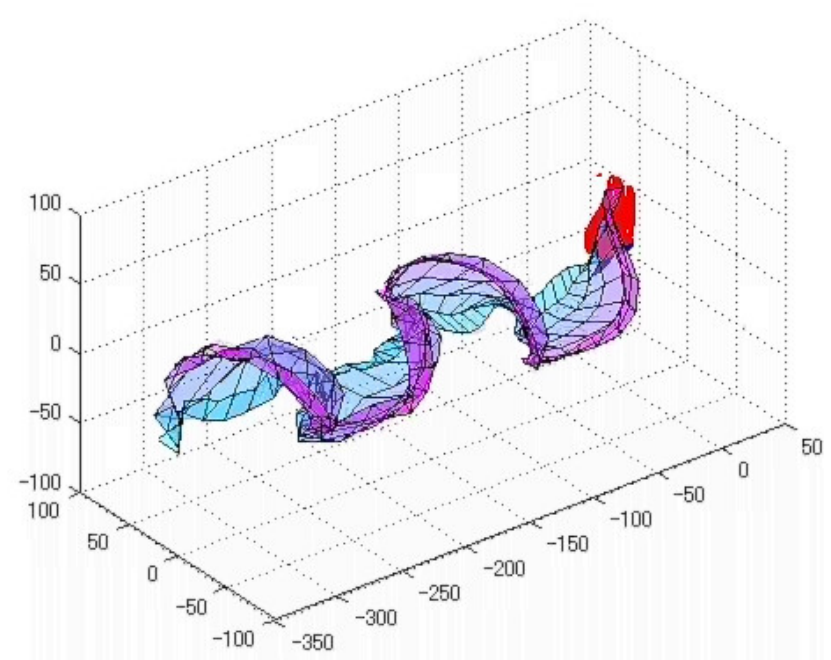

Figure 3. Unsteady wake computed by panel method

flow to the wings is completely unsteady because of the flapping motion, whereas the flow to a cruising fixed-wing aircraft is almost steady. Wing-tip vortices greatly affect the aerodynamic characteristics because a butterfly has almost flat wings of a small aspect ratio. In addition, the flapping motion generates very strong wing-tip vortices. The wing-tip vortices may merge with the free vortices departing from the trailing edge, make complex structures, and greatly influence the aerodynamic characteristics.

Therefore, this study models the aerodynamics of a flapping butterfly using a panel method [19]. Vortex ring element panels are set on wing surfaces and panels with constant strengths of vortices are shed into wakes. Unknown strengths of vortices are determined so as to satisfy the boundary condition of no normal flow across the wing surfaces. The free wake model sheds the panels fulfilling the Kutta condition at trailing edge and the panels move with local stream velocity. This free wake model enables to contain the influence of the unsteady wakes. As a result, this study made a panel method model [3-5].

Fig. 3 shows a wake computed by the panel method for the right wing of the butterfly that is fixed at the thorax in the uniform flow. This figure explains that the necessity for considering the influence of the unsteady wakes.

\subsection{Outline of experimental system}

Verification of the models and parameter setting require fundamental data of flappingof-wings motions and aerodynamic forces. For this purpose, an experimental system with a low-speed wind tunnel is constructed and a wind tunnel experiment is conducted using living butterflies [4].

The butterfly's scientific name is Parantica sita niphonica, which is similar to Danaus in the United States of America. Specifications of the butterfly are listed in Table 1.

The simultaneous measurement system is constructed as illustrated in Fig. 4. 
Table 1. Physical properties of Parantica sita niphonica

\begin{tabular}{clll}
\hline Total mass & $M$ & $\mathrm{~kg}$ & $0.238 \times 10^{-3}$ \\
Aspect ratio & $A R$ & & 4.068 \\
Wing loading & $M g / 2 S_{W}$ & $\mathrm{Nm}^{-2}$ & 0.988 \\
\hline Thorax mass & $m_{B t}$ & $\mathrm{~kg}$ & $0.095 \times 10^{-3}$ \\
Thorax length & $2 l_{B t}$ & $\mathrm{~m}$ & $15 \times 10^{-3}$ \\
Thorax width & $2 w_{B t}$ & $\mathrm{~m}$ & $8 \times 10^{-3}$ \\
\hline Abdomen mass & $m_{B a}$ & $\mathrm{~kg}$ & $0.105 \times 10^{-3}$ \\
Abdomen length & $2 l_{B a}$ & $\mathrm{~m}$ & $20 \times 10^{-3}$ \\
Abdomen width & $w_{B a}$ & $\mathrm{~m}$ & $5 \times 10^{-3}$ \\
\hline Wing mass & $m_{W}$ & $\mathrm{~kg}$ & $0.019 \times 10^{-3}$ \\
Wing length & $y_{t i p}$ & $\mathrm{~m}$ & 0.049 \\
Wing area & $S_{W}$ & $\mathrm{~m}^{2}$ & $0.118 \times 10^{-2}$ \\
\hline
\end{tabular}

Butterfly's motion is measured by the optical measurement system with three high speed cameras that are type FASTCAM-PCI R2 10K of Photoron Ltd. The forces applied to the butterfly are measured by the force measurement system. The butterfly is tethered to the tip of the measure, i.e. the force/torque sensor. The force/torque sensor was made with strain gauges in laboratory. The forces $L$ and $D$, and the moment $M$ corresponding to $\theta_{t}$ are sensed by the force/torque sensor. These forces apply to the force/torque sensor from the butterfly, where $L$ is in vertical upward, $D$ in the mainstream, and $M$ in headup rotation. They include the inertia forces of flapping motion. But, this study refer $L, D$, and $M$ as the lift, the drag, and the pitching moment, respectively. There is a smoke wire for airflow visualization. Motion of a free-flying butterfly is measured by using the experimental system, where measured is the trajectory of flapping flight, i.e. the trajectory of the thorax and joints.

According to calibration experiments, the maximum measuring error in $L$ and $D$ is $0.001[\mathrm{~N}]$ for a range of $\pm 0.02[\mathrm{~N}]$, and that in $M$ is $0.04 \times 10^{-3}[\mathrm{Nm}]$ for $\pm 0.6 \times 10^{-3}[\mathrm{Nm}]$. The cutoff frequency of the measure is $50[\mathrm{~Hz}]$ for noise reduction. The maximum error in position measurements using the video images is $0.13 \times 10^{-3}[\mathrm{~m}]$ in each direction, which is caused by the resolution of images: $0.260 \times 10^{-3}[\mathrm{~m} /$ pixel]. Based on this error, the maximum error in the abdomen angle is $0.37^{\circ}$ for motion measurement. The smoke is made of the propylene glycol by smoke-wire method. According to an evaluation method [20], the tracers in smoke follow-up accurately the air-flow.

\subsection{Experimental observation of motion and force}

Flapping motions and applied aerodynamic forces are measured by wind tunnel experiments using living butterflies [5]. An experiment with Parantica sita niphonica is conducted under a condition that the mainstream velocity is $1.54[\mathrm{~m} / \mathrm{s}]$, the thorax position $x=z=0[\mathrm{~m}]$, and its angle $\theta_{t}=28.1^{\circ}$. Fig. 5 (a) shows the generalized 


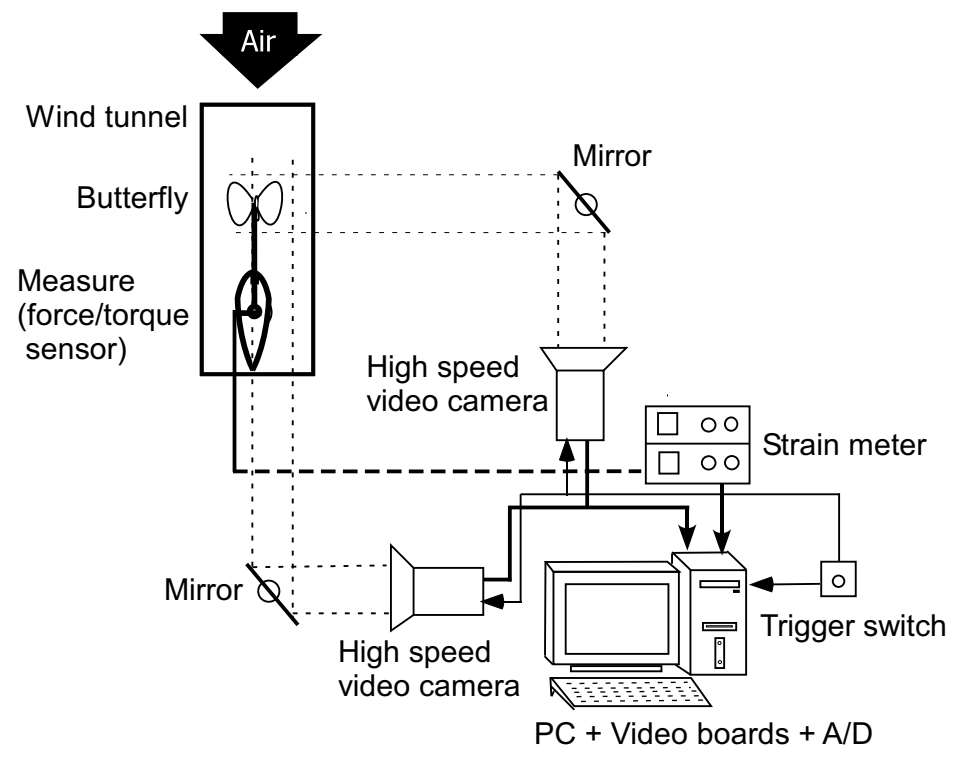

Figure 4. Experimental setup.

coordinates $\boldsymbol{\theta}$ of an experimental result. Figs. 5 (b)-(d) illustrate the measured forces, i.e. the lift $L$, the drag $D$, and the pitching moment $M$. The butterfly repeats this periodic motion in a period of 0.096 seconds during continuous periodic flapping-of-wings motion. Measured aerodynamic forces are also cyclic during the periodic flapping-ofwings motion.

As can be seen in Fig. 5 (a), the flapping angle $\beta$ is a cosine-like curve whose maximum and minimum are $60^{\circ}$ and $-20^{\circ}$. The abdomen angle $\theta_{a}$ is also a sinusoidal curve, but is out of phase to the flapping angle $\beta$. When the butterfly varies amplitude of $\beta$, it changes the minimum of $\beta$ mainly and the maximum marginally. The tethered butterfly swings the abdomen in a larger amplitude than butterflies flying freely. Even if $\beta$ or $\theta_{a}$ changes the amplitude depending on flying conditions and/or individuals, they keep similar smooth cosine curves. Lead-lag angle $\eta$ has a vibration mode whose frequency is twice as large as $\beta$. The feathering angle $\theta$ varies for $30^{\circ}$ from $-10^{\circ}$ to $+20^{\circ}$ through a flapping period. The flapping cycle is split into downstroke and upstroke by the stroke reversal at $t=0.055$ [s]. Fry et al. [21,22] similarly observes fly's flapping-ofwings motion.

This fluctuation of the $\theta$ is considered as a result of a passive wing torsion caused by its structural flexibility since butterflies cannot actively twist their wings in a large angle because of the flapping mechanism. Actually, the torsional torque applied to a wing varies for $0.3 \times 10^{-3}[\mathrm{Nm}]$ and $\theta$ changes for $28^{\circ}$ as the passive torsion that is estimated by using the wing rigidity measured in a later section. In the later section, the passive wing torsion is introduced to the model, and its effect on the flapping-of-wings flight stability is evaluated.

The joint trajectory of the tethered butterfly might be different from that of the free-flying butterfly. Actually, the numerical model of the free-flying butterfly falls down quickly if the measured joint trajectory of the tethered butterfly is used. Therefore, this 
study considers the data obtained by the aerodynamic force measuring experiment as the fundamental data to evaluate the basic model, but does not regard them as the joint trajectory of the free-flying butterfly. Fry et al. [22] discusses the differences of flapping motions of flies between under tethered and free-flying conditions.

\subsection{Verification of mathematical model}

The wing on each side is divided by ten panels in the span direction and four panels in the cord direction. There are panels as the free-vortices in wakes for two flapping periods. The butterfly flaps for two periods before simulation starts in order to converge the flow field induced by the free-vortices in the wakes. The number of panels in the cord direction is chosen so as Katz and Plotkin [19] recommended. In typical numerical simulations, instantaneous aerodynamic forces at any moment change up to $1 \%$ of the averaged forces even if other numbers increase. The thorax trajectory is changed less.

The motion of the butterfly is solved by the modified Euler method that has the second order accuracy, where the sampling time is $T / 1000$ and $T$ is a flapping period. New panels of wakes depart from the wing trailing edges every $T / 20$. The period $T / 20$ is used because the differences of trajectories are less than the differences caused by the above-mentioned wake length and so on. Hence, numerical results, e.g., the thorax trajectory, converge. The obtained results agree well with the following experimental results.

The $\boldsymbol{\theta}$ in Fig. 5 (a) obtained from the experiment and its derivatives $\dot{\boldsymbol{\theta}}$ and $\ddot{\boldsymbol{\theta}}$ are substituted into the mathematical models of the butterfly that is tethered on the back of the thorax. Its aerodynamic forces $\boldsymbol{\tau}_{\text {air }}$ are illustrated in Figs. 5 (b)-(d). The forces $L, D$, and $M$ in both of the experiment and the simulation include the inertia forces of flapping motion. In case of $L$, the inertial force is up to $20 \%$ of the maxmimum of $L$. The average values of $L, D$, and $M$ per flapping cycle are $0.3 \times 10^{-3}[\mathrm{~N}], 1.8 \times 10^{-3}[\mathrm{~N}], 0.14 \times 10^{-3}[\mathrm{Nm}]$ in the experiment and $0.7 \times 10^{-3}[\mathrm{~N}], 1.3 \times 10^{-3}[\mathrm{~N}], 0.04 \times 10^{-3}[\mathrm{Nm}]$ in the simulation. Validity of the model can be evaluated by comparing the simulation results with the experimental data. The panel method model can calculate the aerodynamic forces with the same degree of accuracy for any experiment and agree well with them though it tends to estimate the lift being a little smaller than the experiment results. There are rarely met other researches [23] that report numerical results in good agreement with experimental measurements of small insects like this study.

Left figures in Fig. 6 show that the flow around the wings visualized by a smoke wire. From the experimental result, it is understood that the complex flowfield is formed by the flapping-induced free vortex in wakes. In numerical simulations of the panel method, the velocity at any point can be obtained by Biot-Savart law, which calculates the velocity induced by each vortex element. In right figures, small arrows at lattice points denote the local velocities relative to mainstream calculated by the panel method. In depth direction, the arrows are illustrated simultaneously from $-0.12[\mathrm{~m}]$ to $0[\mathrm{~m}]$. In the figures, more long arrows appear at a region with larger induced velocity. These figures 


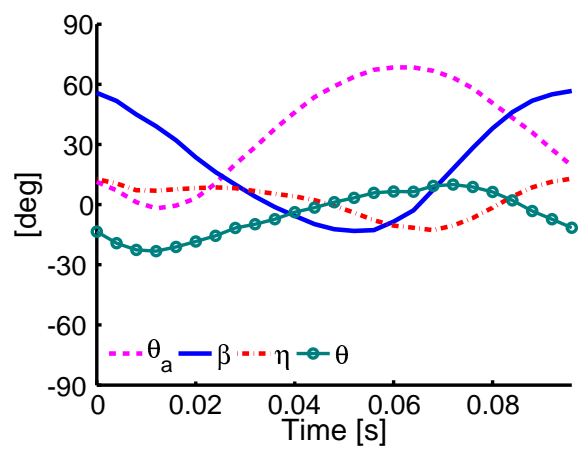

(a) Motion

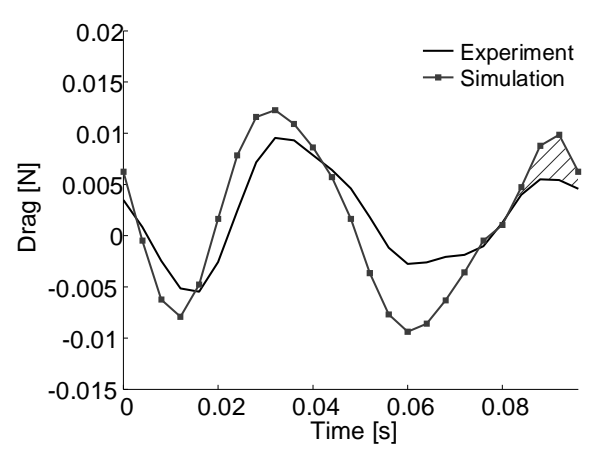

(c) Drag

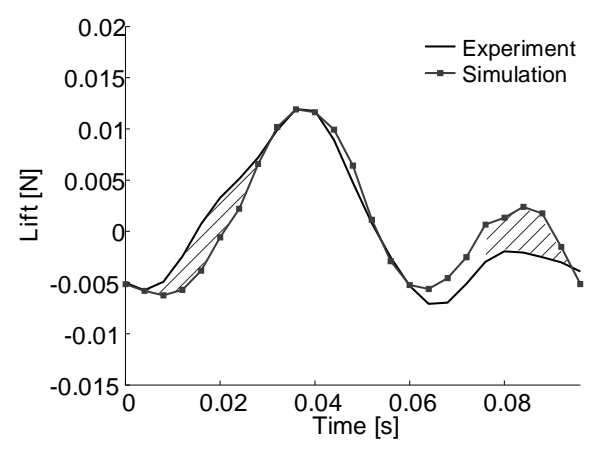

(b) Lift

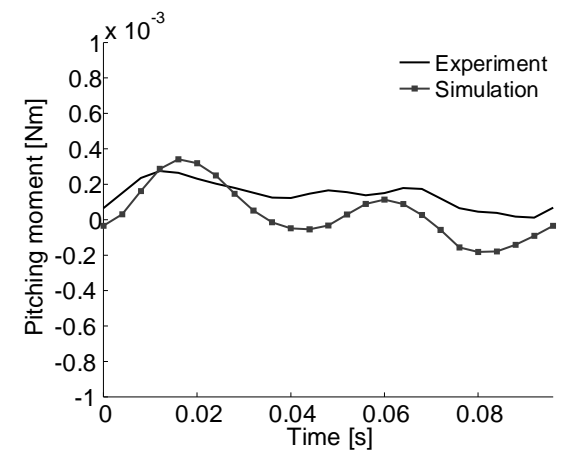

(d) Pitching moment

Figure 5. Motion and aerodynamic forces of experiment and panel method model.

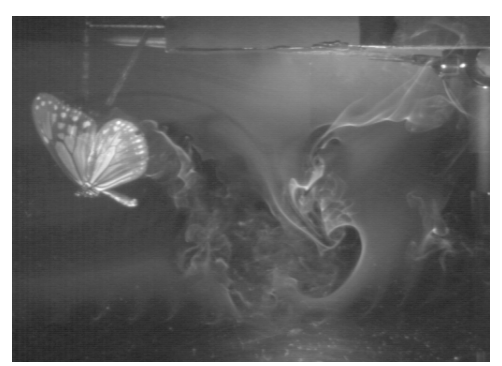

(a) Experiment

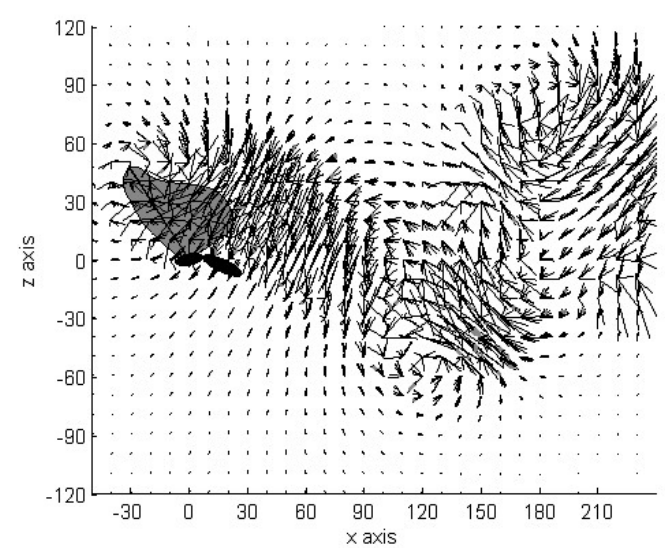

(b) Simulation

Figure 6. Comparison of flow fields of experimental visualization and numerical simulation by panel method at the beginning of downstroke. $(V=1.0[\mathrm{~m} / \mathrm{s}]$, $\left.\theta_{t}=-10^{\circ}\right)$

are suitable to compare with the experimental images qualitatively. This numerical simulation captures the unstedy flowfield of the experiment.

Viscosity effect should be considered in the model because of the low Reynolds number $R e \simeq 10^{3}$ for the butterfly. However, both computational and experimental evaluations show that the viscous drag is negligible amount with approximately $0.5 \%$ of the maximum drag. The viscous drag is measured when a butterfly is stationary 
and its wings are parallel to the mainstream. Analytical computation for this situation supports the experimental measurement. Compressibility is not taken into account because of low speed. In addition, it is observed by the smoke wire visualization that the flow passes along wing surfaces during wingbeat $[1,2]$. Hence, by assuming inviscid incompressible flow without separation, a panel method based on the potential flow is applied in this study. The mean wing-tip velocity and the maximum wing-tip velocity are $4 C_{\beta} f y_{\text {tip }}=1.2[\mathrm{~m} / \mathrm{s}]$ and $2 \pi C_{\beta} f y_{\text {tip }}=1.9[\mathrm{~m} / \mathrm{s}]$, respectively, where $C_{\beta}, f, y_{\text {tip }}$ are the amplitude of $\beta$, the frequency of flapping, and the wing-tip length, respectively. The maximal wing-tip velocity is larger than the velocity of the main stream $u_{0}=1.54[\mathrm{~m} / \mathrm{s}]$. It indicates that the maximal wing-tip velocity is larger than the traveling velocity of the butterfly. The Reynolds number estimated by the mean wing-tip velocity and the wing-tip length is $R e=4 C_{\beta} f y_{\text {tip }}^{2} / \nu_{\text {air }}=4800$. The Strouhal number in the experiment is $S t=4 C_{\beta} f y_{\text {tip }} / u_{0}=0.80$. The Froude number is $F r=4 C_{\beta} f \sqrt{y_{\text {tip }} / g}=1.6$.

Motion of a free-flying butterfly is measured by using the experimental system of Figure 4. Figure 7 (a) and (b), respectively, show a period of the measured entire motion of the periodic free-flight and a numerical result where the entire motion is calculated by the model using the measured joint trajectory. Figure 7 (c) shows the measured joint trajectory. The flapping cycle is split into downstroke and upstroke by the stroke reversal at $t=0.06[\mathrm{~s}]$. The numerical result duplicates the free flight, even though the final thorax angles are different. The other existing models constructed by the present authors cannot duplicate the free-flight, i.e. the simple method [1], the lumped-vortex method considering characteristic flapping-of-wings effects [2], and the panel method model without free-vortices in wakes [2]. The numerical result duplicates the free-flight considering flow field induced by the free-vortices in wakes.

In addition, the flapping flight of this modeled butterfly is unstable. [5] Hence, the trajectory of the model is necessarily different from that of the living butterfly because there exist some errors in modeling, initial condition, etc. It has not been reported that another numerical simulation using a joint trajectory of a living butterfly duplicates its periodic free flight. Therefore, obtained model is relatively accurate.

\section{Periodic flapping flight of model}

The difference of the entire free-flying motions and that of the aerodynamic forces in Fig. 5 represent some dynamics differences of the model and the actual butterfly. It shows that the model does not perfectly reproduce the entire free-flying motion of the actual butterfly, even though the measured joint trajectory of an actual butterfly is used. Hence, a flapping joint trajectory is parametrically searched below for a periodic free flight of the numerical model. 


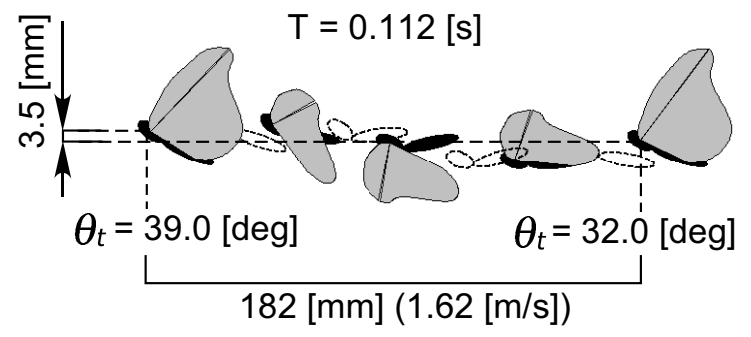

(a) Experimental measurement

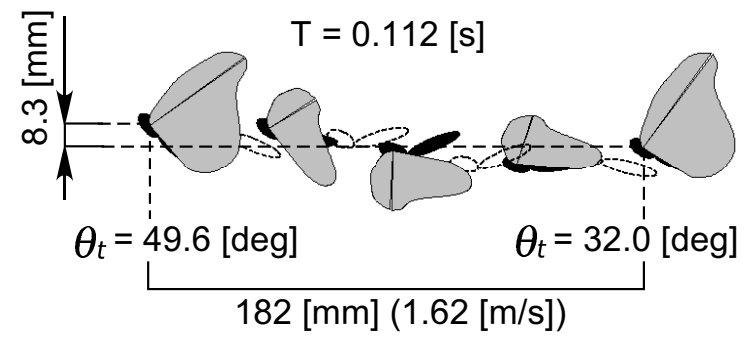

(b) Numerical simulation

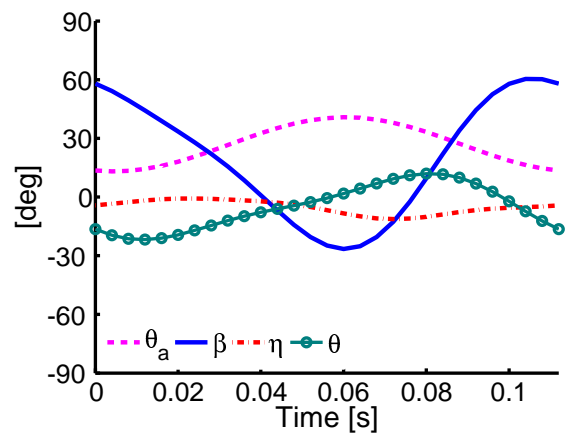

(c) Joint trajectory

Figure 7. Measured free-flying of flapping butterfly and numerical simulation using measured joint motion

\subsection{Trajectory search method}

The periodic flapping-of-wings flight is defined as "all other than $x$ in state vector $\boldsymbol{\theta}_{f}$ and $\dot{\boldsymbol{\theta}}_{f}$ after a flapping period $T$ agree with initial value $\boldsymbol{\theta}_{s}$ and $\dot{\boldsymbol{\theta}}_{s}$."

Joint angle trajectories are described by Fourier series for periodic motions. Fourier parameters and initial conditions are considered as learning parameters of vector $\boldsymbol{w}$ to find a trajectory of the periodic flapping-of-wings flight. In order to reduce computation efforts, the number of parameters is cut down based on the knowledge from the experiments and observation.

The following cost function $J$ is used for the learning evaluation:

$$
J=\sum_{i} Q_{i}\left(\theta_{f i}-\theta_{s i}\right)^{2}+R\left\{E\left(\boldsymbol{\theta}_{f}, \dot{\boldsymbol{\theta}}_{f}\right)-E\left(\boldsymbol{\theta}_{s}, \dot{\boldsymbol{\theta}}_{s}\right)+\sum_{j} \int_{0}^{T} \tau_{j} \mathrm{~d} \theta_{j}\right\}
$$

where $E(\boldsymbol{\theta}, \dot{\boldsymbol{\theta}})=T(\boldsymbol{\theta}, \dot{\boldsymbol{\theta}})+V(\boldsymbol{\theta})$ is the mechanical energy with the kinetic energy $T(\boldsymbol{\theta}, \dot{\boldsymbol{\theta}})$ and the potential energy $V(\boldsymbol{\theta})$. The $\theta_{j}$ and $\tau_{j}$ are the angle and the torque 
of joint $j$. The $Q_{i}$ and $R$ are evaluation weights. The suffix $i$ denotes one of $z, \theta_{t}$, $\dot{x}, \dot{z}$ or $\dot{\theta}_{t}$. The suffix $j$ also denotes one of $\theta_{a}, \beta, \eta$ or $\theta$. The first term of Eq. (6) evaluates the periodicity of motion by the quadratic form of error between boundaries. The second term of Eq. (6) is mechanical energy loss during the period. One obtains the most efficient flapping-of-wings flight for a mathematical model by minimizing the cost function. A gradient method finds the above-mentioned learning parameters such that the obtained trajectory satisfies the definition of the periodic flapping-of-wings flight. The obtained trajectories are evaluated by comparing with experimental results.

\subsection{Searched trajectories}

The panel method model can obtain almost periodic trajectories, though they do not perfectly correspond to the definition of the periodic flight. One of the obtained flights is illustrated in Fig. 8. Figures (a), (b), and (c) are the searched flapping motion, the searched joint trajectory, and the joint trajectory measured by the experiment, respectively. The searched joint trajectory (b) is similar to the measured joint trajectory (c). There are some different joint trajectories that realize almost periodic trajectories. On the other hand, joint trajectories of free-flights measured from living butterflies are more versatile. The peak and bottom of the flapping angle $\beta$ are approximately $90^{\circ}$ and $-60^{\circ}$ in the numerical simulation and $90^{\circ}$ and $-70^{\circ}$ in the experiment. Those of the abdomen angle $\theta_{a}$ are approximately $60^{\circ}$ and $-60^{\circ}$ in the numerical simulation and $60^{\circ}$ and $-70^{\circ}$ in the experiment. The peak and bottom positions of time are almost same. The differences of amplitudes of $\beta$ and $\theta_{a}$ are approximately $6 \%$ and $8 \%$, respectively. The differences have almost same dynamical effects as those of inertia because a butterfly change the abdomen mass during experiments.

The butterfly can fly for several periods when one of those motions is just repeated, whereas no feedback control is applied. It is reported that the unsteady wake-induced flow has a type of feedback stabilization effect that enables butterfly to fly several periods $[3,5]$, whereas the details are not mentioned in this paper. This is the feedback stabilization effect brought by this system through the dynamic characteristic of the flow field, and it can be considered as an implicit control.

The solid line with open squares in Fig. 15 (a) illustrates that the flapping flight of the model is leaving gradually from the initial flight and destabilized after 4 flapping periods. It is also seen in Fig. 15 (a) that the oscillation of thorax causes the instability of the flight though the mathematical model precisely repeat the desired joint motion by a controller for numerical simulations.

\section{Effect of wing flexibility on aerodynamic forces}

The wing deformations of free-flying butterflies were qualitatively shown in some photographs [24] and sketches [17], whereas quantitative measurements and discussions [15, 25-30] have been limited yet. Experimental measurements of wing 


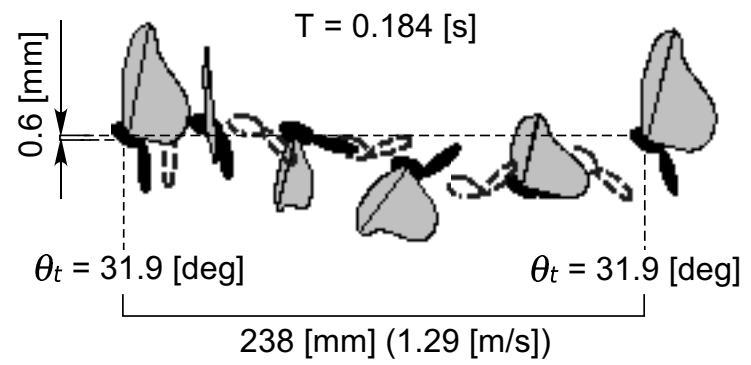

(a) Almost periodic flight obtained by numerical simulation

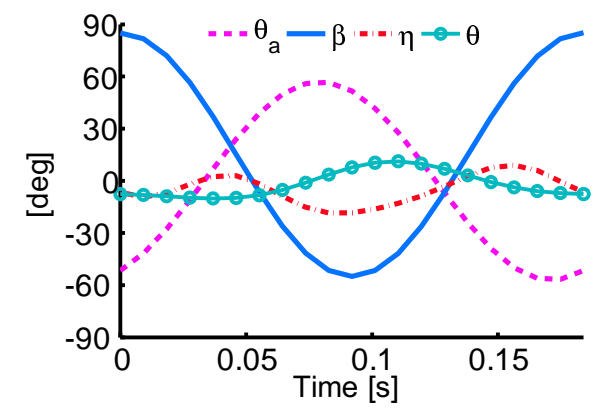

(b) Joint trajectory obtained by numerical simulation

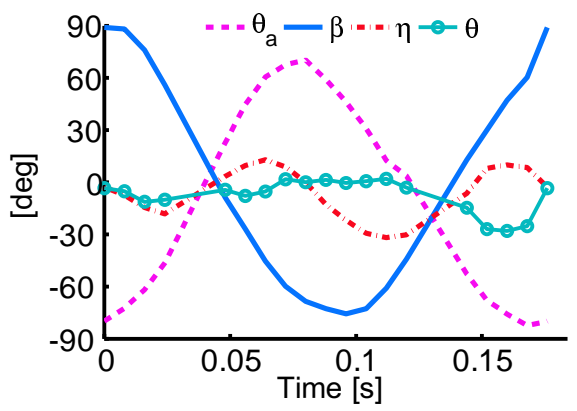

(c) Joint trajectory measured by experiment

Figure 8. Almost periodic flapping-of-wings flight of panel method model with flat wings and its original joint trajectory measured by experiment

kinematics and wing structural characteristics have been conducted mainly for the tethered insects. Wang et al. [31] measures wing deformation of a free-flying dragonfly using a sophisticated equipment with a seated laser light and high-speed video cameras. They captures the deformation roughly because precise and accurate measurement is not available. A large field of view is needed because a free-flying insect changes its position, but it results in a low resolution and inaccurate measurements.

Three high-speed video cameras take synchronized images to maintain measurement accuracy. A wing model with time-varying camber is introduced into the basic model, where the parameters to represent the deformation are determined at each time so that the outer shape of the model agrees with the outline of video images. This is a robust 3-dimensional reconstruction method as well as the strip method of Willmott et al. $[25,26]$ whereas the wing deformation model used in this method is different from the strip method. Walker et al. $[9,10]$ measured the deformation of wings of a desert 
locust, whereas deformations of butterfly wings have not known in detail.

Structurally flexible wings of actual butterflies are passively deformed by inertia force and aerodynamic forces applied to the wings. The above model is called the timevarying camber model that describes the measured deformation as a function of time. This time-varying camber is introduced into the basic model, and the aerodynamic forces are calculated. The time-varying camber model is compared with the basic model whose wings are always flat. The aerodynamic effects of the wing deformation is quantified by using the experimental observation and the models. It will be shown that some good effects are provided by the wing deformations.

\subsection{Observation wing deformation}

Video images of a flapping butterfly are taken to observe the bending of wings. Figs. 9 and 10 show the images from three directions.

When aerodynamic forces are applied, wings are deformed as follows:

(i) At the beginning of the downstroke $(t=0.008[\mathrm{~s}])$, the overlapping right-and-left wings are peeled from the leading edges with elastic deformation and become convex upward. It is known as the peel mechanism [13].

(ii) During the downstroke $(t=0.024,0.040,0.056[\mathrm{~s}])$, wings keep being convex upward.

(iii) In transition between the downstroke and the upstroke $(t=0.080[\mathrm{~s}])$, wings are almost flat.

(iv) During upstroke $(t=0.104[\mathrm{~s}])$, wings are convex downward on the contrary.

On each side, the forewing and the hindwing almost always move together as a single wing and do not flap separately. Therefore, two wings on each side are considered as a single wing and the time-varying camber will be modeled later for the combined wing.

This experiment is chosen because the wing deformations are easy to observe, whereas this is different from Fig. 5. The characteristic wing deformations are observed commonly in other experiments including that of Fig. 5. In the parts with hatching in Fig. 5, numerical simulations often have large differences from experiments. The wings are convex upward in the beginning part, and they are convex downward in the latter. The wings deform largely in the both parts.

In the simulation, the difference should be caused since the butterfly is modeled by the rigid wings in the simulation. Therefore, the structural flexibility of wings is needed to be modeled and evaluate the effects.

\subsection{Wing model with time-varying camber}

The modeled wings which can bend are shown in lower rows in Figs. 9 and 10. and the modeled wings successfully capture the deformations of the real wings. The shapes of the wings are given as a function of time that is observed in the experiment, where the reason will be described later. 
Effects of Structural Flexibility of Wings in Flapping Flight of Butterfly
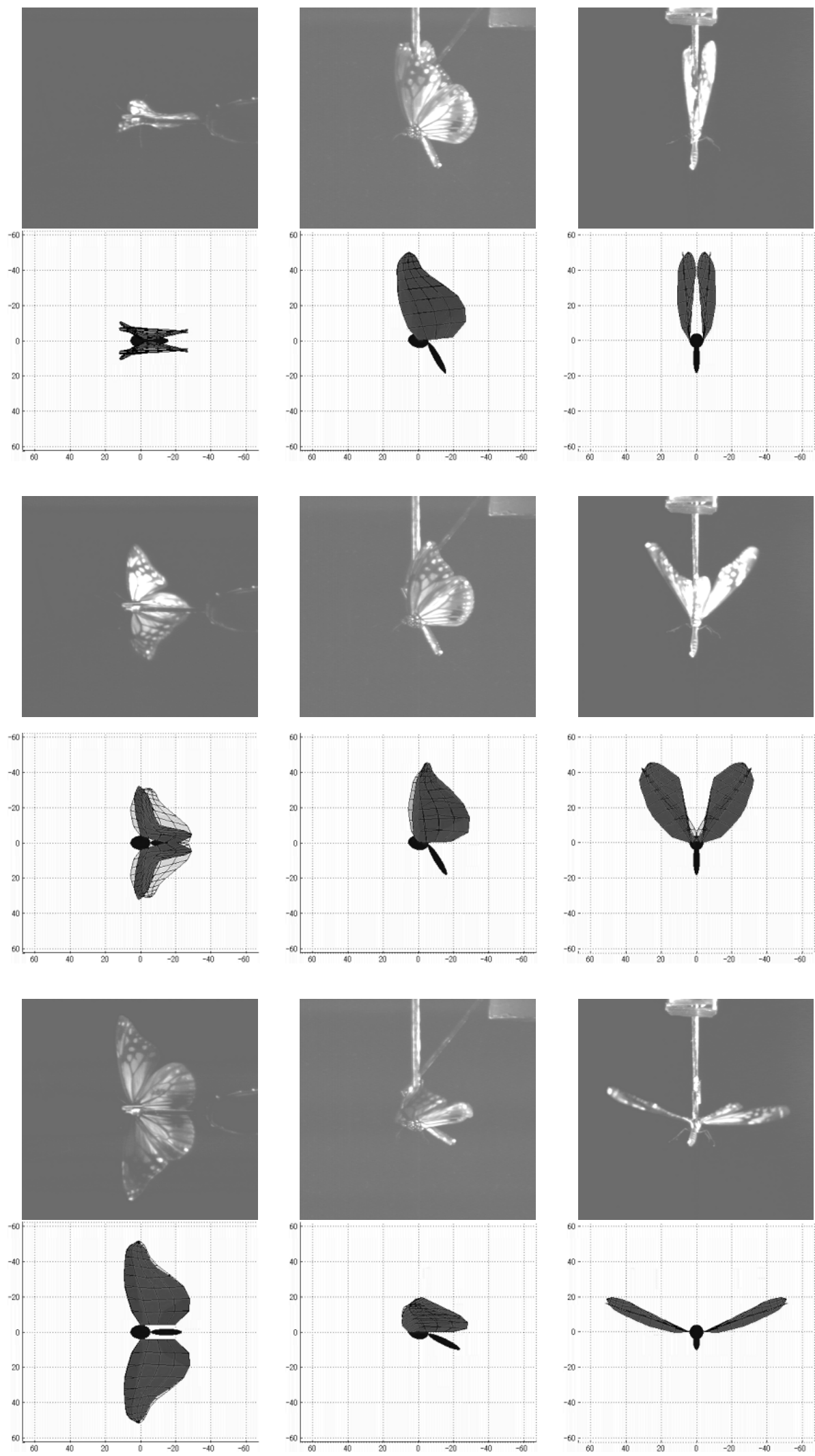

Figure 9. Wing deformations of experiment and model (top: $t=0.008[\mathrm{~s}]$, middle: $t=0.024[\mathrm{~s}]$, bottom: $t=0.040[\mathrm{~s}])$ 
Effects of Structural Flexibility of Wings in Flapping Flight of Butterfly
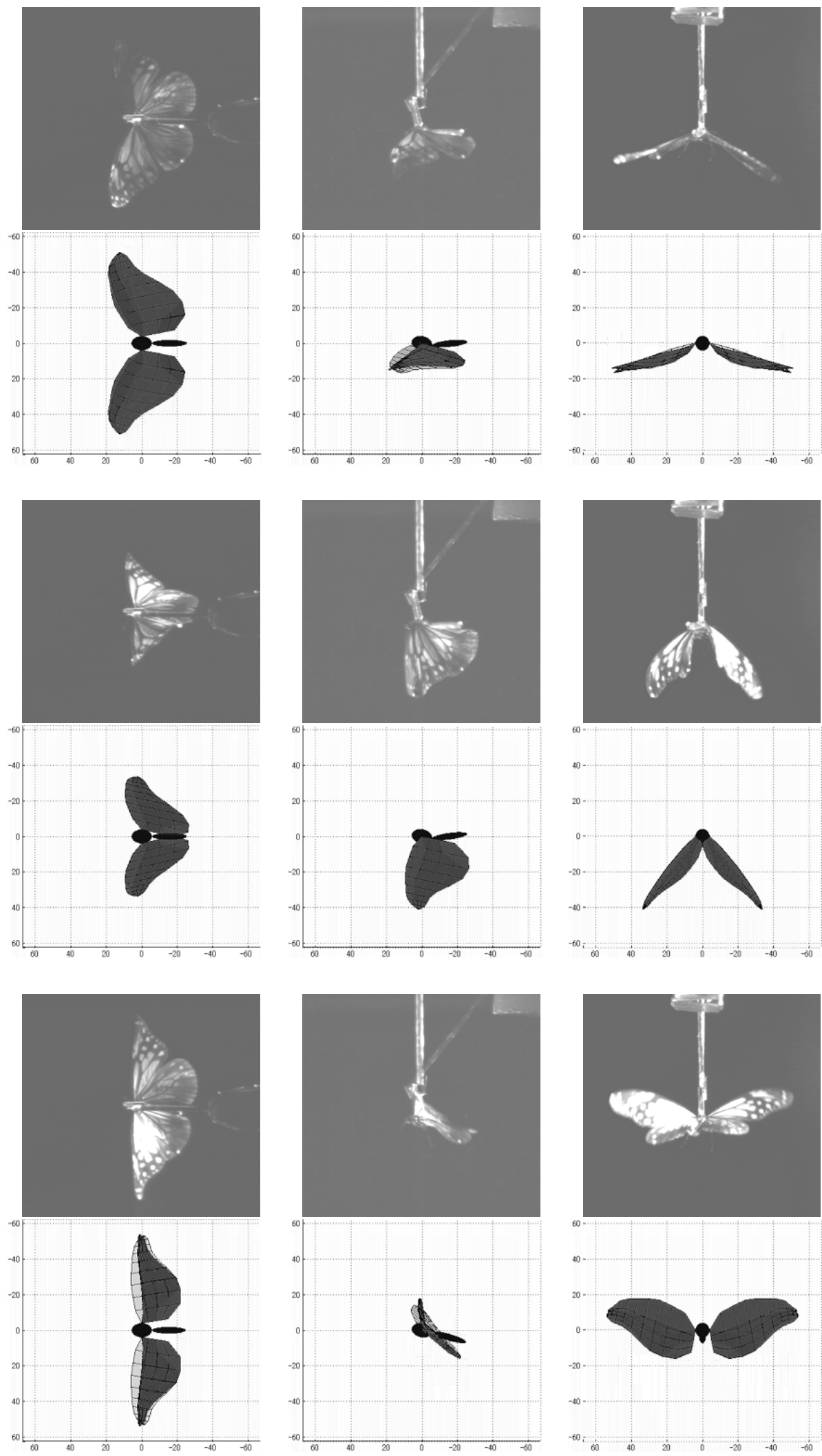

Figure 10. Wing deformations of experiment and model (top: $t=0.056$ [s], middle: $t=0.080[\mathrm{~s}]$, bottom: $t=0.104[\mathrm{~s}])$ 


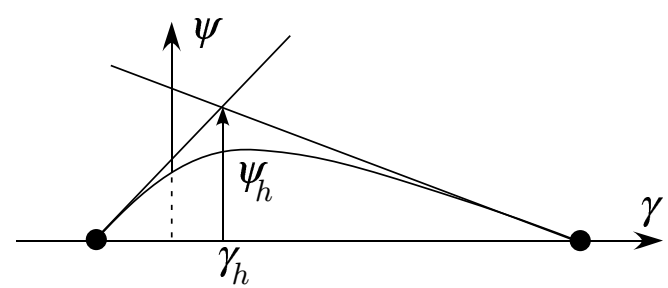

Figure 11. Airfoil function $\psi_{h}$ defined by two lines

As shown in Fig. 11, the airfoil is defined as a hyperbolic curve $\psi_{h}$ that has two tangent lines. At a time, all of the front side tangent lines along span direction are on a same flat plane. The rear side tangent lines also form a flat plane at a time. Hence, the wing configuration is defined by two flat planes, i.e. the front side plane and the rear side plane. By using video images of the experiment, the front side plane and the rear side plane are determined to be tangent at the leading edge of forewing and at the hind wing, respectively.

The wing configuration is determined as a function of time, where the parameters defining the front side plane and the rear side plane are given as functions of time. In the panel method, vortex panels are placed on the wings whose configurations are defined above.

\subsection{Effects on aerodynamic forces caused by wing deformation}

Fig. 12 shows the aerodynamic forces of the experiment, the panel method model with flat wings, and that with time-varying camber wings. The average values of $L, D$, and $M$ per flapping cycle are $2.4 \times 10^{-3}[\mathrm{~N}],-2.5 \times 10^{-3}[\mathrm{~N}],-0.056 \times 10^{-3}[\mathrm{Nm}]$ in the experiment, $0.3 \times 10^{-3}[\mathrm{~N}],-2.9 \times 10^{-3}[\mathrm{~N}],-0.006 \times 10^{-3}[\mathrm{Nm}]$ in the the panel method model with flat wings, $1.1 \times 10^{-3}[\mathrm{~N}],-3.5 \times 10^{-3}[\mathrm{~N}],-0.013 \times 10^{-3}[\mathrm{Nm}]$ in the model with time-varying camber wings. In terms of the lift, the bending wing model generates larger lift than the flat wing model during the downstroke. The lift typically increases by the upward convex of wings during the downstroke. In terms of the drag, the bending wing model generates less drag than the flat wing model at the latter half of the upstroke. It is because of the downward convex of wings during the upstroke. As a result, the bending wing model is closer to the experiment than the flat wing model.

The typical effects of the deformation caused by the structural flexibility are organized as follows:

a It begins immediately after beginning of downstrokes that the wings become convex upward by the peel mechanism.

b During downstrokes, the wings keep being convex upward, which improve the aerodynamic efficiency as increasing the lift and decreasing the drag.

The following improvements of the aerodynamic efficiency are observed by comparing the experiment with the numerical simulation with bending wings, whereas the numerical simulation has small discrepancies from the experiment. 


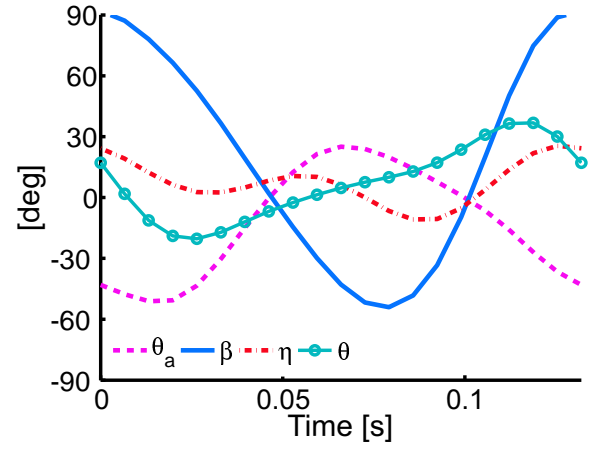

(a) Motion

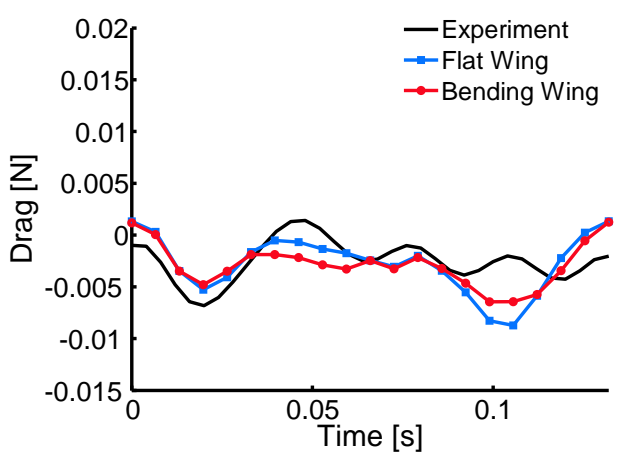

(c) Drag

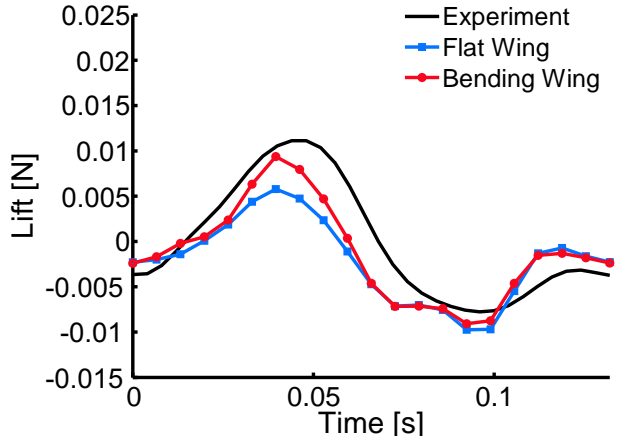

(b) Lift

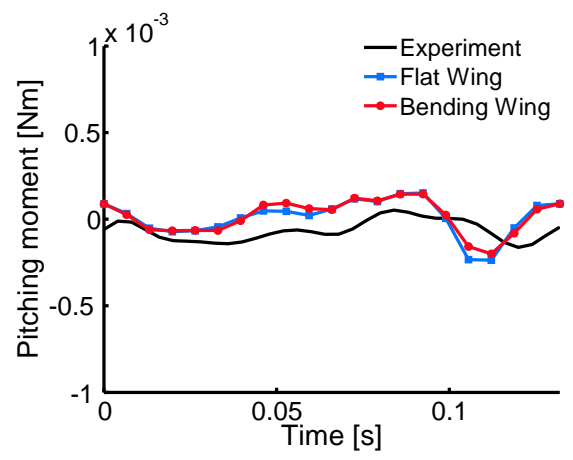

(d) Pitching moment

Figure 12. Motion and aerodynamic forces of experiment, of numerical simulation with flat wings, and of numerical simulation with bending wings.

c At the beginning of downstrokes, the experimental peel mechanism generates larger thrust.

d During downstrokes, the experimental wings convex upward generate larger lift.

Following reasons can be considered why the panel method model with bending wings has the quantitative error. The closer aerodynamic forces to the experiment are calculated, the more accurate the wing deformation is given. For the self-consistency of physical phenomena, the wing deformation should be obtained as the elastic deformation caused by the aerodynamic forces, whereas the deformation has been given by the function of time from the experimental observation. It will be a future subject because a precise measurement of the rigidity distribution is more difficult than the deformation measurement. Both the local loads and the deformations should be measured precisely all over the wings for the rigidity distribution.

\section{Effect of wing flexibility on flighi dynamics}

In this section, only torsion is considered as the wing deformation but it is obtained as the elastic deformation caused by the aerodynamic forces, while the bending has been given by the function of time in the previous section 3 . This model is called a passive torsion wing model. 


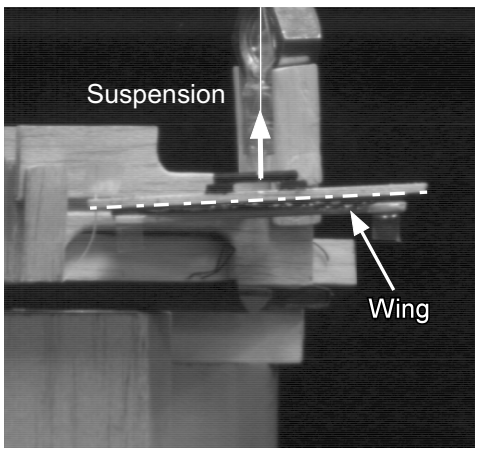

(a) Without torsion

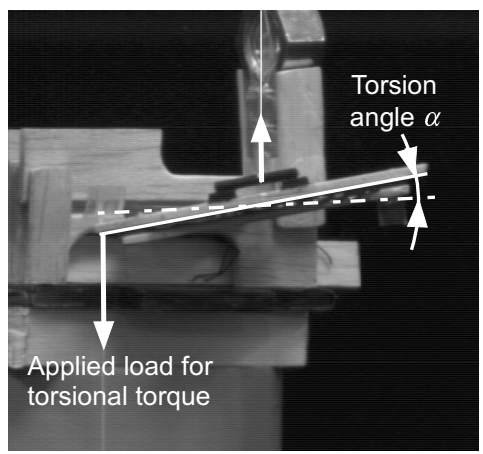

(b) With torsion

Figure 13. Side view images of experiments to measure wing torsional rigidity.

As discussed in Fig. 5 (a), deformation of wings is observed in experiments, and the structural flexibility leads to large torsion of wings. Flapping motion of the model is controlled to follow the desired trajectory without error in section 3 , whereas that of actual butterflies cannot be controlled in this way because their wings are twisted by various unexpected disturbances. This passive torsion of wings has not been considered in the simulation of section 3. In this section, wing torsion due to the structural flexibility is introduced to the model and its effect on the flapping-of-wings flight is examined.

\subsection{Experiments to measure wing rigidity}

To introduce the passive torsion to the mathematical model, the torsional rigidity of the wings has been measured with living butterflies. In this experiment, three typical-size butterflies are chosen. Both wings of three butterflies are used to measure rigidity.

Torsional torque loads are applied to a wing of living butterfly, where the root of the wing is clamped to fix it to the horizontal plane. The torsional angle $\alpha$ is measured when torsional torque load $\tau$ is applied, where the angles are captured from camera images. Pure torsional torque is applied by using a jig so that the deformation of wings becomes twisting only. The distance of the load point from the fixed wing root is half of wing length. Fig. 13 shows camera images of the experiments. No torque is applied on the wing in Fig. 13 (a), an appropriate torque is applied on the wing in Fig. 13 (b), and the difference of angles is measured as the torsional angle.

The torsional rigidity $K$ of the wing is then given by $K=\tau / \alpha$ with respect to the torsional torque loads. The torsional rigidity of wings as the average of the living butterflies is approximately $6.0 \times 10^{-4}[\mathrm{Nm} / \mathrm{rad}]$. The damping ratio is estimated as 0.7 from the wing motion, and it is applied to the model.

\subsection{Modeling of flexibly torsional wings}

Fig. 14 shows a 1-DOF damped oscillator model of wing torsion caused by its structural flexibility, where a spring and a damper are installed in the joint at the wing root. The model of the wing remains as a flat plate. 


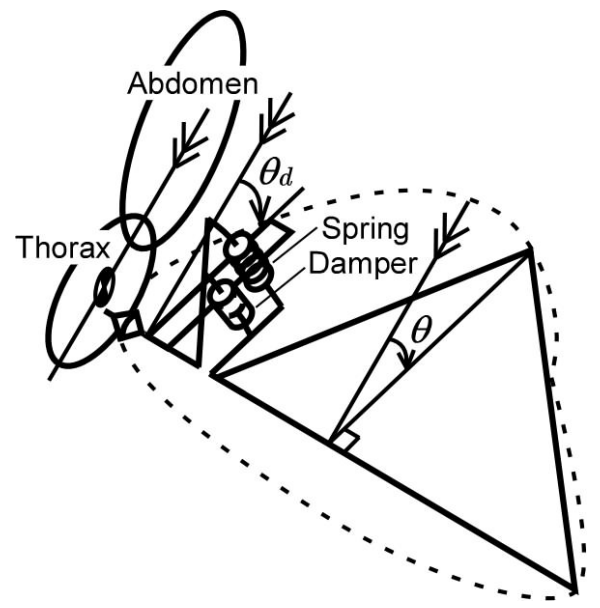

Figure 14. One-DOF damped oscillator model of wing torsion

This modeling defines the joint torsion torque $\tau_{\theta}$ that is the generalized force of the feathering angle representing a torsion angle $\theta$ as:

$$
\begin{aligned}
\tau_{\theta} & =\tilde{\tau}_{\theta}+\tau_{\theta, \text { flex }} \\
& =\tilde{\tau}_{\theta}-G_{P, \theta}\left(\theta-\theta_{d}\right)-G_{D, \theta}\left(\dot{\theta}-\dot{\theta}_{d}\right)
\end{aligned}
$$

where $\tilde{\tau}_{\theta}$ is the feedforward torque for the desired feathering angle $\theta_{d}$, and $\tau_{\theta, \text { flex }}$ is the feedback torque when the $\theta$ has error from $\theta_{d}$. This torque models the structural flexibility of the wing. Feedback gain $G_{P, \theta}$ corresponds to the torsional rigidity of the wing and $G_{P, \theta}=K$. The flapping motions of the models with and without flexibly torsional wings are the same if the joint trajectories of the two models have no difference. Hence, the desired trajectory $\theta_{d}$ is commonly used for the two models. Feedback gain $G_{D, \theta}$ is a damping coefficient corresponds to the torsional damping of the wing.

\subsection{Effect of wing torsion on flapping-of-wings flight}

Numerical simulations show how the flexible torsion changes the flight stability using mathematical model with measured flexibility of wings. Figs. 15 (a), (b), and (c) show two trajectories of flapping-of-wings flights. The two trajectories have been obtained using the models with and without flexibly torsional wings, where the same desired joint trajectories are applied to the both models. The desired joint trajectory has been obtained by the trajectory searching for the rigid model in section 3 . It is seen that the oscillation of the thorax causes the instability of the flight for the model without flexibly torsional wings. The passive torsion wings extend the stable flight for four more periods. Fig. 15 (d) shows that the oscillation of $\ddot{\theta}_{t}$ is reduced, especially when the butterfly without the structural flexibility is going down at $t=0.8[\mathrm{~s}]$. The joint trajectory of the model without flexibility before falling down is almost same as that of the model with flexibility. The slight errors in joint motions are caused by the difference in joint dynamics. The wing flexibly damps the pitching oscillation of the thorax, where the oscillation may make the butterfly fall down. As the result, the instability of the thorax 


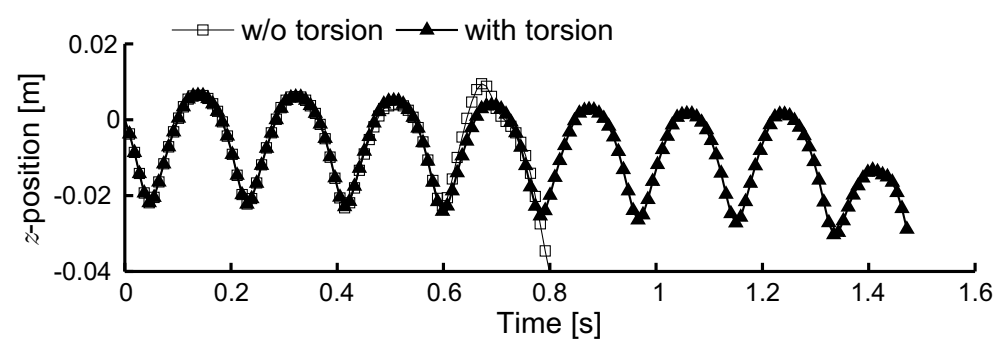

(a) Height

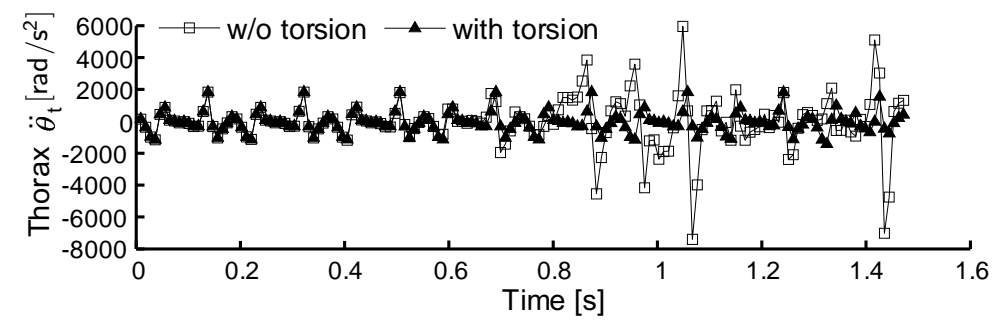

(b) Acceleration of pitching of thorax

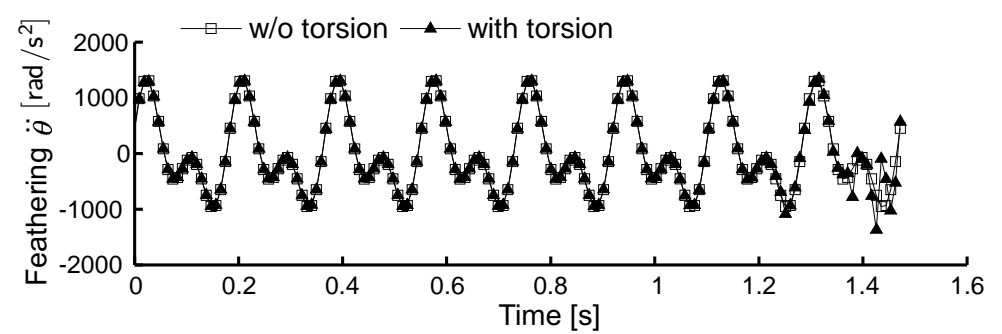

(c) Acceleration of feathering angle

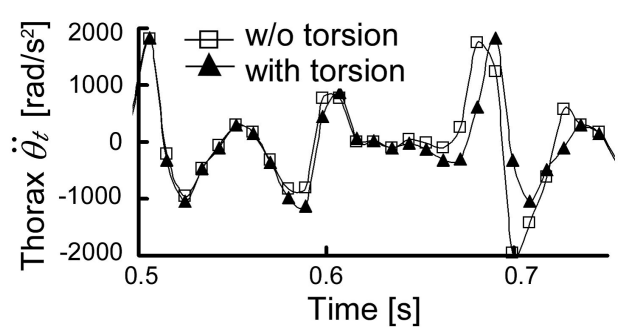

(d) Acceleration of pitching of thorax $(0.5 \leq t \leq 0.75[\mathrm{~s}])$

Figure 15. Flapping-of-wings flights with/without flexible torsion.

is reduced, and the flying periods are extended.

The same stabilizing effects are demonstrated in three of four other simulations by the passive wing torsion in the mathematical model, where flapping motion is different from each case. One of them is illustrated in Fig. 16. Therefore, the flexible torsion introduces the stabilization effect on the flapping-of-wings flight.

This is a kind of feedback stabilization effect brought by this system through the dynamic property of the wing. It can be considered as a kind of preflex [32] as well as an implicit control. 


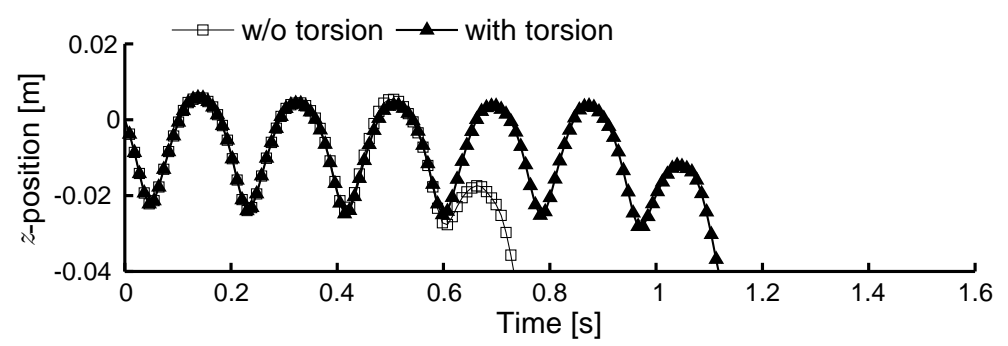

(a) Height

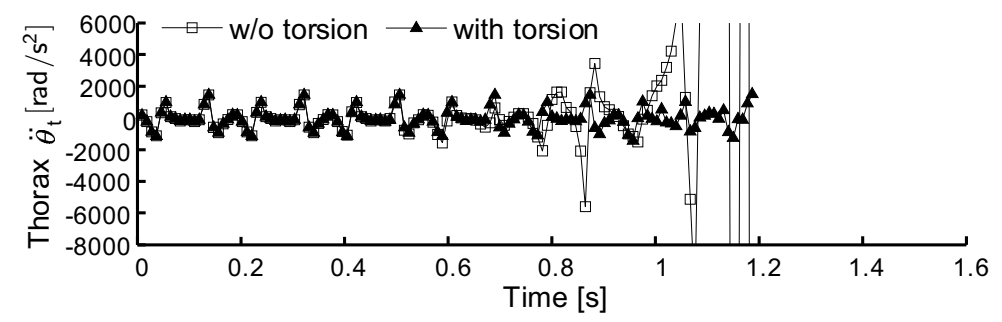

(b) Acceleration of pitching of thorax

Figure 16. Other flapping-of-wings flights with/without flexible torsion.

\section{Concluding Remarks}

To discuss effects of the structural flexibility of the wings of butterfly in flapping-of-wings flights, the butterflies are modeled. The panel method has been applied to calculate the aerodynamic forces. The flapping-of-wings flight obtained from the parametric search of the trajectories is unstable with the thoracic oscillation. Two models are proposed to integrate two aspects of the wing deformation. One model, in which the bending is modeled, has clarified the effect on the aerodynamic forces. In the other model, passive wing torsion caused by structural flexibility is modeled. In this passive torsion model, the flat wings are passively twisted due to the aerodynamic disturbance. Using this model, effects of passive torsion on flight dynamics are discussed.

The flight instability has been decreased by introducing the passive wing torsion in the second model. The living butterflies have the structurally flexible wings that improve both the aerodynamic efficiency and the flight stability.

\section{Acknowledgments}

A part of this work has been financially supported by a Grant-in-Aid for Scientific Research from Ministry of Education, Science, Culture, and Sports of Japan.

\section{References}

[1] K. Senda, T. Tanaka, and M. Sawamoto. Measurement and numerical simulation of a flapping butterfl. In Proceedings of 2nd International Symposium on Adaptive Motion of Animals and Machines, pages 1-7, WeP-II-1, Kyoto, mar 2003. 
[2] K. Senda, M. Sawamoto, T. Shibahara, and T. Tanaka. Study on flapping-of-wings flight of butterfly with experimental measurement. In Proceedings of AIAA Atmospheric Flight Mechanics Conference, pages 1-14, AIAA Paper 2004-5368, Reston, Virginia, aug 2004. AIAA.

[3] K. Senda, M. Sawamoto, T. Tanaka, and T. Shibahara. Analysis on control of flapping-of-wings flight of butterfly. In Proceedings of 3rd International Symposium on Adaptive Motion of Animals and Machines, pages 1-12, Ilmenau, Germany, sep 2005.

[4] K. Senda, M. Sawamoto, T. Shibahara, M. Kitamura, and T. Tanaka. Study on flappingof-wings flight of butterfly with numerical and experimental analysis. In Proceedings of AIAA Atmospheric Flight Mechanics Conference, pages 1-16, AIAA Paper 2006-6150, Reston, Virginia, aug 2006. AIAA.

[5] K. Senda, M. Sawamoto, M. Kitamura, and T. Tanaka. Stabilization of flapping-of-wing flight of butterfly, considering wakes. In N. Kato and S. Kamimura, editors, Bio-mechanisms of Swimming and Flying, pages 193-204. Springer, Tokyo, 2007.

[6] K. Senda, M. Sawamoto, M. Kitamura, and T. Obara. Towards realization of stable flapping-ofwings flight of butterfly. In Proceedings of 4th International Symposium on Adaptive Motion of Animals and Machines, pages 62-63, Cleveland, Ohio, USA, jun 2008.

[7] K. Senda, T. Obara, M. Kitamura, and T. Nishikata. On flight mechanics of flapping butterfly. In Workshop on Future Trends of Mobiligence: in IEEE International Conference on Robotics and Automation, pages 17-22, Kobe, Japan, may 2009.

[8] M. Vanella, T. Fitzgerald, S. Preidikman, E. Balaras, and B. Balachandran. Influence of flexibility on the aerodynamic performance of a hovering wing. J. Exp. Biol., 212:95-105, 2009.

[9] S. M. Walker, A. L. R. Thomas, and G. K. Taylor. Photogrammetric reconstruction of highresolution surface topographies and deformable wing kinematics of tethered locusts and freeflying hoverflies. Journal of the Royal Society, Interface, 6(33):351-366, 2009.

[10] S. M. Walker, A. L. R. Thomas, and G. K. Taylor. Deformable wing kinematics in the desert locust: how and why do camber, twist and topography vary through the stroke? Journal of the Royal Society, Interface, 6(38):735-747, 2009.

[11] J. Young, S. M. Walker, R. J. Bomphrey, G. K. Taylor, and A. L. R. Thomas. Details of insect wing design and deformation enhance aerodynamic function and flight efficiency. Science, 325:1549$1552,2009$.

[12] H. Tanaka and I. Shimoyama. Forward flight of swallowtail butterfly with simple flapping motion. Bioinspiration and Biomimetics, 5:026003, 2010.

[13] C. P. Ellington. The aerodynamics of flapping animal flight. Integrative and Comparative Biology, 24(1):95-105, 1984.

[14] R. Dudley and C. P. Ellington. Mechanics of forward flight in bumblebees: I. kinematics and morphology. Journal of Experimental Biology, 148:19-52, 1990.

[15] J. M. Wakeling and C. P. Ellington. Dragonfly flight: Ii. velocities, accelerations and kinematics of flapping flight. Journal of Experimental Biology, 200:557-582, 1997.

[16] G. K. Taylor and R. Zbikowski. Nonlinear time-periodic models of the longitudinal flight dynamics of desert locusts schistocerca gregaria. Journal of the Royal Society, Interface, 2(3):197-221, 2005.

[17] A. K. Brodsky. The Evolution of Insect Flight. Oxford University Press, Oxford, 1994.

[18] A. Azuma. The Biokinetics of Flying and Swimming. AIAA, Reston, Virginia, 2nd edition, 2006.

[19] J. Katz and A. Plotkin. Low-Speed Aerodynamics. Cambridge University Press, Cambridge, 2nd edition, 2001.

[20] The Visualization Sociaty of Japan. Handbook of Particle Image Velocimetry. Morikita Publishing Co., Ltd., Tokyo, 2002.

[21] S. N. Fry, R. Sayaman, and M. H. Dickinson. The aerodynamics of free-flight maneuvers in drosophila. Science, 300:495-498, 2003.

[22] S. N. Fry, R. Sayaman, and M. H. Dickinson. The aerodynamics of hovering flight in drosophila. Journal of Experimental Biology, 208:2303-2318, 2005. 
[23] H. Aono, F. Liang, and H. Liu. Near- and far-field aerodynamics in insect hovering flight: An integrated computational study. Journal of Experimental Biology, 211:239-257, 2008.

[24] S. Dalton. Caught in Motion. John Calmann and Cooper, London, UK, 1986.

[25] A. P. Willmott and C. P. Ellington. Measuring the angle of attack of beating insect wings: Robust three-dimensional reconstruction from two-dimensional images. Journal of Experimental Biology, 200:2693-2704, 1997.

[26] A. P. Willmott and C. P. Ellington. The mechanics of flight in the hawkmoth manduca sexta: I. kinematics of hovering and forward flight. Journal of Experimental Biology, 200:2705-2722, 1997.

[27] R. J. Wootton. Leading edge section and asymmetric twisting in the wings of flying butterflies. Journal of Experimental Biology, 180:105-117, 1993.

[28] S. J. Steppan. Flexural stiffness patterns of butterfly wings. Journal of Research on the Lepidoptera, 35:61-77, 2000.

[29] S. A. Combers and L. T. Daniel. Flexural stiffness in insect wings: I. scaling and the influence of wing venation. Journal of Experimental Biology, 206:2979-2987, 2003.

[30] S. A. Combers and L. T. Daniel. Flexural stiffness in insect wings: Ii. spatial distribution and dynamic wing bending. Journal of Experimental Biology, 206:2989-2997, 2003.

[31] H. Wang, L. Zeng, H. Liu, and C. Yin. Measuring wing kinematics, flight trajectory and body attitude during forward flight and turning maneuvers in dragonflies. Journal of Experimental Biology, 206:745-757, 2003.

[32] I. E. Brown and G. E. Loeb. A reductionist approach to creating and using neuromuscular models. In J. M. Winters and P. E. Crago, editors, Biomechanics and Neural Control of Posture and Movement. Springer, New York, 2000. 